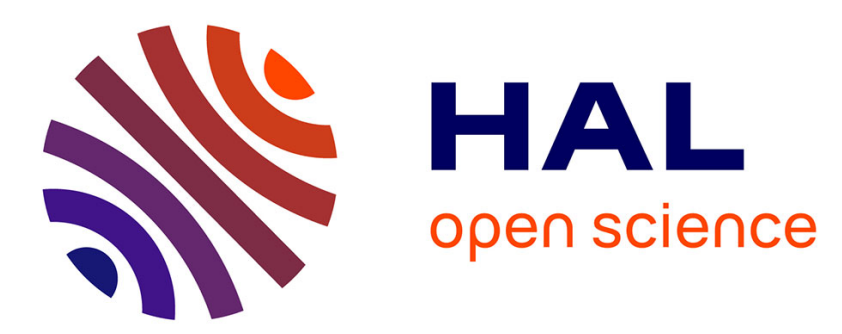

\title{
Étude d'un compteur de neutrons à efficacité élevée et à réponse définie dans la bande d'énergie 0-14 Mev
}

\author{
A. Picot, M. Lebars, R. Haug
}

\section{To cite this version:}

A. Picot, M. Lebars, R. Haug. Étude d'un compteur de neutrons à efficacité élevée et à réponse définie dans la bande d'énergie 0-14 Mev. Revue de Physique Appliquée, 1967, 2 (3), pp.218-224. 10.1051/rphysap:0196700203021800 . jpa-00242794

\section{HAL Id: jpa-00242794 https://hal.science/jpa-00242794}

Submitted on 1 Jan 1967

HAL is a multi-disciplinary open access archive for the deposit and dissemination of scientific research documents, whether they are published or not. The documents may come from teaching and research institutions in France or abroad, or from public or private research centers.
L'archive ouverte pluridisciplinaire HAL, est destinée au dépôt et à la diffusion de documents scientifiques de niveau recherche, publiés ou non, émanant des établissements d'enseignement et de recherche français ou étrangers, des laboratoires publics ou privés. 


\title{
ÉTUDE D'UN GOMPTEUR DE NEUTRONS \\ A EFFIGAGITÉ ÉLEVÉE ET A RÉPONSE DÉFINIE DANS LA BANDE D’ÉNERGIE 0-14 MeV
}

\author{
Par A. PICOT, M. LEBARS, R. HAUG, \\ Laboratoire de Physique Théorique et Nucléaire de la Faculté des Sciences de Nancy.
}

\begin{abstract}
Résumé. - Un compteur de neutrons dont l'efficacité varie de manière connue entre 30 et $10 \%$ dans la bande d'énergie $0-14 \mathrm{MeV}$ est étudié. Il fournit une mesure approximative de l'énergie du rayonnement d'une source quelconque et il permet son étalonnage à environ $10 \%$ près. Ce compteur a été étalonné avec 5 sources neutroniques de nature différente. Des courbes d'étalonnage ont été établies par une étude théorique du compteur et elles ont été confirmées par les résultats expérimentaux.
\end{abstract}

Abstract. - A neutron counter whose efficiency varies between 30 and $10 \%$ within the energy range $0-14 \mathrm{MeV}$ is studied. It gives an approximate radiation energy measurement and an activity calibration with $10 \%$ accuracy. Calibration curves have been established by a theoretical study of the counter and they have been verified by experimental results.

Introduction. - Nous avons réalisé et étalonné un compteur à efficacité connue permettant l'étalonnage rapide de sources de neutrons dont les énergies sont comprises dans la bande $0-14 \mathrm{MeV}$. Il est constitué par un bloc de paraffine cylindrique dans lequel sont placés, parallèlement à l'axe, douze détecteurs au bore répartis en deux couronnes indépendantes $\left(r_{1}=7 \mathrm{~cm}, r_{2}=12 \mathrm{~cm}\right)$. Chaque couronne peut être reliée à un ensemble électronique indépendant. L'intérêt de ce compteur est dû au fait que, pour une source donnée, le rapport des taux de comptage de ces deux couronnes $n_{\mathrm{e}} / n_{\mathrm{i}}$ est une fonction croissante de l'énergie entre 0 et $14 \mathrm{MeV}$ (courbe 2, fig. 6).

$\mathrm{Si}$ la source est monoénergétique, on déduit son énergie de la mesure du rapport $n_{\mathrm{e}} / n_{\mathrm{i}}$; à cette énergie correspond sur la figure 6 , courbe 1 , une efficacité donnée du compteur.

Si la source n'est pas monoénergétique, l'énergie liée au rapport $n_{\mathrm{e}} / n_{\mathrm{i}}$ permet encore de déterminer l'efficacité réelle du compteur relativement à cette source, à condition que les efficacités des couronnes extérieure et intérieure, $\varepsilon_{\theta}$ et $\varepsilon_{\mathrm{i}}$, soient liées par une relation de la forme :

$$
\varepsilon_{\theta}(E)=A \varepsilon_{\mathrm{i}}(E)+B
$$

$A$ et $B$ étant des constantes propres du compteur.

Dans le cas de notre compteur, cette propriété n'est vérifiée qu'entre 4 et $14 \mathrm{MeV}$ seulement, ce qui permet néanmoins l'étalonnage de la plupart des sources usuelles. La précision est de l'ordre de $10 \%$ et ne dépend que de l'exactitude des courbes d'étalonnage.
Le compteur, sous sa forme actuelle, présente néanmoins l'avantage de la simplicité de mise en ouvre et de la précision relativement bonne qu'il est susceptible de fournir dans l'étude de sources dont le spectre énergétique est inconnu. Il permet d'étudier des sources neutroniques de très faible activité (inférieure à $1 \mathrm{n} / \mathrm{s}$ ), des émissions neutroniques multiples (corps spontanément fissiles) et il est peu sensible au rayonnement gamma.

I. Description et réalisation du compteur. - 1 . MILIEU RALENTISSEUR. - La qualité d'un détecteur de neutrons de grande efficacité est liée au choix du milieu ralentisseur pour les neutrons rapides et on sait que ce sont les milieux hydrogénés denses qui conviennent le mieux. Il est possible de détecter les neutrons rapides par leur proton de recul [1], [2] ou de les ralentir avant de les compter. Nous avons adopté la deuxième solution [3], mais elle limite l'efficacité puisque, d'une part, tous les neutrons ne sont pas thermalisés dans le bloc ralentisseur qui a nécessairement des dimensions limitées et, d'autre part, tous les neutrons thermiques ne sont pas capturés par les éléments détecteurs, l'hydrogène du milieu réagissant également.

2. Dimensions du blog ralentisseur. Étude du FLUX THERMIQUE EN MILIEU INFINI. - Les dimensions ont été choisies après une expérience qui nous a permis de faire un compromis entre un bloc ralentisseur où seraient thermalisés tous les neutrons, mais encombrant, et un bloc de petites dimensions, donc 


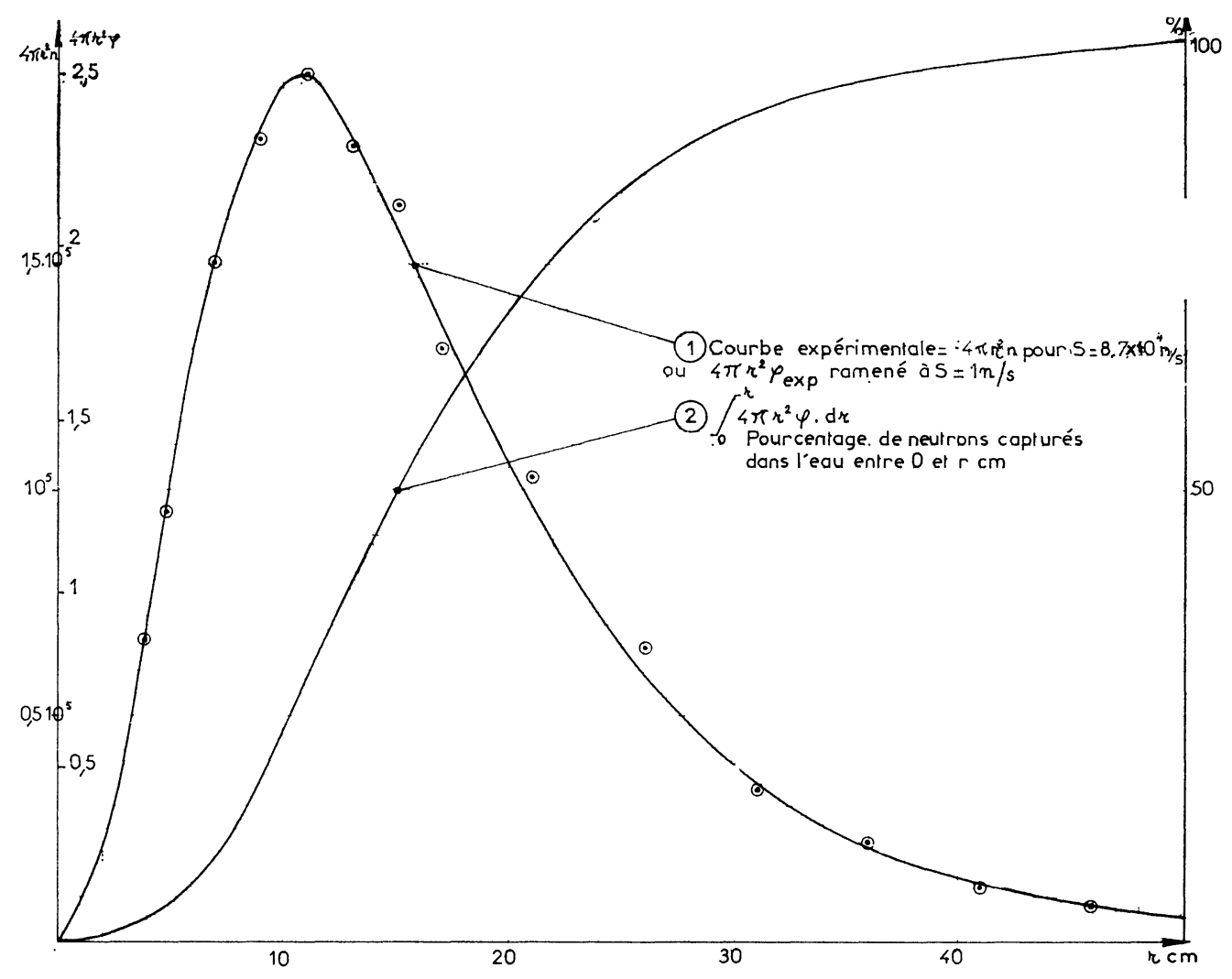

FIG. 1. - Variation du flux thermique

à la distance $r$ d'un point source (PoBe) de neutrons rapides en géométrie infinie dans l'eau.

peu efficace. Cette expérience consiste à déplacer un petit détecteur au trifluorure de bore (LCT 01 NE 3/1) dans une grande cuve remplie d'eau $(2 \times 3 \times 1,5 \mathrm{~m})$ au centre de laquelle est immergée une source de neutrons, et à mesurer la variation du flux thermique en fonction de la distance (courbe 1, fig. 1). Cette expérience a deux conséquences :

D'une part, pour la source utilisée (PoBe), elle donne la proportion de neutrons thermalisés entre 0 et $r$, soit $90 \%$ entre 0 et $30 \mathrm{~cm}$ (courbe 2, fig. 1) et $70 \%$ entre 0 et $20 \mathrm{~cm}$.

D'autre part, elle permet de vérifier l'exactitude des prévisions théoriques concernant la variation du flux en fonction de la distance (10), ce qui permet d'étendre à d'autres sources, d'énergie différente, les résultats obtenus pour cette source particulière. La théorie prévoit que la proportion de neutrons thermalisés entre 0 et $20 \mathrm{~cm}$ est de $100 \%$ pour des neutrons de $0 \mathrm{MeV}$ et de $30 \%$ pour des neutrons de $14 \mathrm{MeV}$.

Finalement, nous avons choisi un cylindre de paraffine de $50 \mathrm{~cm}$ de haut, $20 \mathrm{~cm}$ de rayon. Nous avons vérifié que la perte d'Albédo due aux couches de rayon supérieur à $20 \mathrm{~cm}$ était faible, la proportion de neutrons piégés dans le ralentisseur étant encore de $65 \%$ pour la source de PoBe.
3. Nature et position des DÉtecteurs. - Nous avons utilisé des détecteurs au trifluorure de bore de type LCT BE $40 / 5$ de $40 \mathrm{~cm}$ de long et $5 \mathrm{~cm}$ de diamètre qui présentent une bonne efficacité pour les neutrons thermiques.

Une étude préalable faite dans une cuve identique au bloc ralentisseur mais contenant de l'eau [5] montre que, en déplaçant les détecteurs, l'efficacité maximale pour la source $\mathrm{PoBe}$, avec 8 détecteurs, est de $21 \%$. Avec 12, elle atteint $24 \%$ et la disposition géométrique des détecteurs influe peu sur l'efficacité.

De plus, un arrangement des 12 détecteurs en deux couronnes contenant respectivement 4 et 8 détecteurs (rayons 7 et $12 \mathrm{~cm}$ ) fournit une efficacité de $23 \%$ et présente l'avantage d'améliorer la protection contre les sources extérieures. En rassemblant les résultats précédents, on observe que la proportion de neutrons piégés dans le bloc ralentisseur varie entre 100 et $30 \%$ (pour des énergies comprises entre 0 et $14 \mathrm{MeV}$ ); mais comme la proportion de neutrons capturés par le bore est sensiblement constante et voisine de $30 \%$, il s'ensuit que l'efficacité globale doit varier entre 30 et $10 \%$ dans la limite d'énergie comprise entre 0 et $14 \mathrm{MeV}$. 
4. Description du Gompteur et de son ÉlegtroNIQUE. - La disposition des 12 détecteurs est représentée sur la figure $2 a$. L'appareillage électronique utilisé ( fig. $2 b$ ) est classique (un préamplificateur de
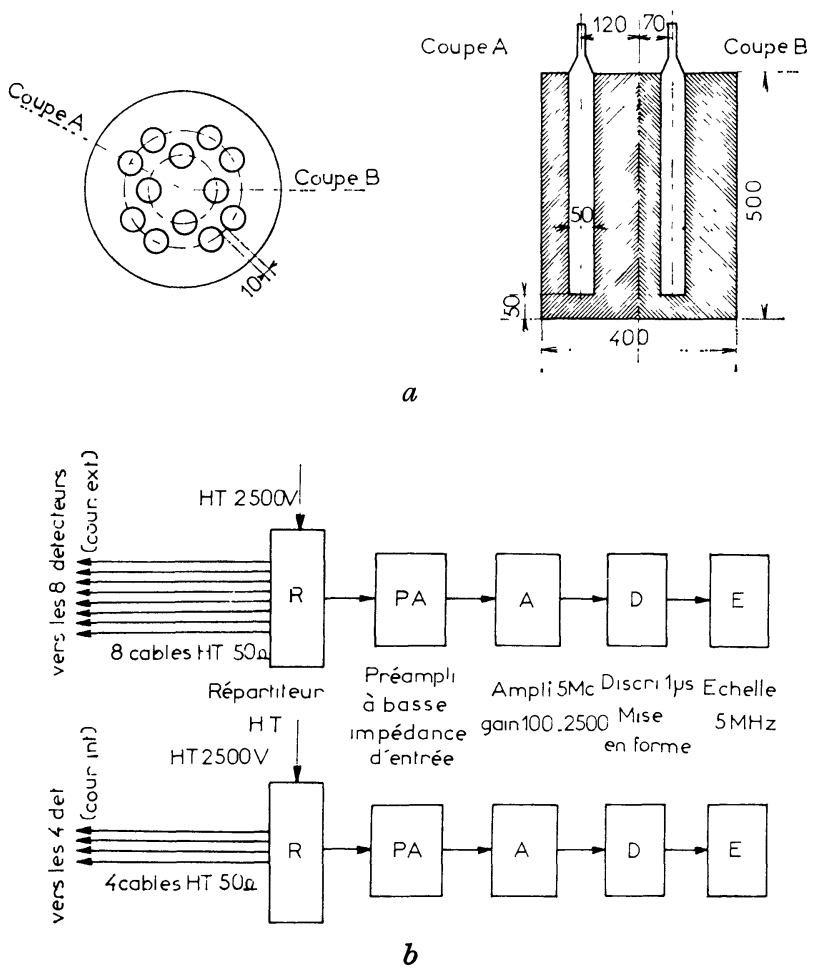

FIG. 2.

charge permettant de brancher un ou plusieurs détecteurs sans modifier les réglages) [6]; il comporte une chaîne de comptage séparée pour chaque couronne.

Nous avons vérifié la bonne reproductibilité des résultats avec un compteur géométriquement semblable. De plus, nous avons constaté que l'efficacité de chaque détecteur d'une même couronne était la même à $5 \%$ près, et que la position du pic $\alpha$ issu de chaque détecteur restait la même à $1 \%$ près.

II. Etalonnage du compteur en fonction de l'énergie. Mesure du rapport des taux de comptage des couronnes extérieure et intérieure. - En toute rigueur, il faudrait disposer, pour étalonner ce compteur, de sources monoénergétiques. L'utilisation de neutrons fournis par les réactions nucléaires $(d, d)$ et $(d, t)$ répond à ce critère et c'est la raison pour laquelle elles ont été utilisées. Certaines sources produisant des neutrons par réaction $(\gamma, \mathrm{n})$ sont également monoénergétiques et c'est pourquoi nous avons utilisé une source antimoine-béryllium produisant des neutrons de $24 \mathrm{keV}$ ( $\pm 3 \mathrm{keV})$. D'autre part, nous disposions de sources de polonium-fluor et poloniumbéryllium pour lesquelles le spectre est donné dans la littérature [4]; on peut donc définir une énergie moyenne par la relation :

$$
E_{\mathrm{m}}=\frac{\int E \mathrm{~d} n}{\int \mathrm{d} n}
$$

$\mathrm{d} n(E)$ étant le nombre de neutrons d'énergie comprise entre $E$ et $E+\mathrm{d} E$.

Les sources utilisées et les énergies moyennes correspondantes sont représentées dans le tableau suivant; notons aussi que nous avons établi le spectre de la source de PoBe et nous avons obtenu un résultat en bon accord avec celui donné dans la référence [4].

\begin{tabular}{ccc} 
Sources & Énergie & MoYenNe \\
- & \multicolumn{3}{c}{ - } \\
(d, t) & 14 & $\mathrm{MeV}$ \\
$(\mathrm{d}, \mathrm{d})$ & 2,4 & $\mathrm{MeV}$ \\
Sb-Be & $0,025 \mathrm{MeV}$ \\
Po-F & 1 à $2 \mathrm{MeV}$ \\
Po-Be & 5 & $\mathrm{MeV}$
\end{tabular}

L'énergie moyenne étant connue, il faut encore déterminer l'activité de ces sources, ce qui nécessite divers étalonnages que nous allons maintenant examiner.

1. ÉtAlonnage des sources. - En ce qui concerne les sources $(d, d)$ et $(d, t)$, l'étalonnage peut facilement être résolu par comptage des particules associées et nous examinerons en détail le montage expérimental dans l'étude du compteur.

Pour les autres sources, nous avons procédé par comparaison avec un étalon d'activité connue. Pour des raisons pratiques, nous avons choisi une source étalon de type polonium-fluor dont nous pouvons vérifier l'activité donnée par le constructeur.

A. Étalonnage d'une source PoF par coïncidence $\gamma-\gamma$ [7]. - Dans une source du type polonium-fluor, deux rayons gamma d'annihilation étant émis en coïncidence, on peut relier l'activité gamma $n(t)$ à l'activité neutronique $S(t)$ à la date $t$ de la mesure, la date 0 étant celle de fabrication de la source :

$$
\begin{aligned}
n(t)=S_{0} \frac{\lambda_{2}}{\lambda_{1}-\lambda_{2}}\left(\mathrm{e}^{-\lambda_{2} t}-\right. & \left.\mathrm{e}^{-\lambda_{1} t}\right) \\
& =0,173 S_{0}\left(\mathrm{e}^{-\lambda_{3} t}-\mathrm{e}^{-\lambda_{1} t}\right)
\end{aligned}
$$

$S_{0}$ étant l'activité initiale; $\lambda_{1}$ et $\lambda_{2}$ sont les constantes radioactives du polonium et du sodium 22 .

Une expérience de coïncidences permet de mesurer l'activité gamma, la mesure correcte nécessite l'utilisation d'une source de référence de sodium 22 pour éliminer l'influence des rayonnements $\gamma$ parasites de la source de neutrons.

La mesure donne :

$$
S_{0}=4,32 \times 10^{4} \text { neutrons } / \mathrm{s} \text { à } 2 \% \text { près }
$$

valeur qui est en bon accord avec celle fournie par le constructeur (Radio Chemical Centre) :

$$
S_{0}=4,31 \times 10^{4} \text { neutrons } / \mathrm{s} \text { à } 2 \% \text { près. }
$$


B. Étalonnage des sources $\mathrm{PoBe}, \mathrm{SbBe}$ par activation de sulfate de manganèse [8]. - Nous avons utilisé la source PoF pour calibrer un compteur à activation de sulfate de manganèse, ce qui nous a permis de mesurer l'activité des autres sources $\mathrm{PoBe}$ et $\mathrm{SbBe}$. L'appareillage est représenté figure 3 . La source de neutrons à

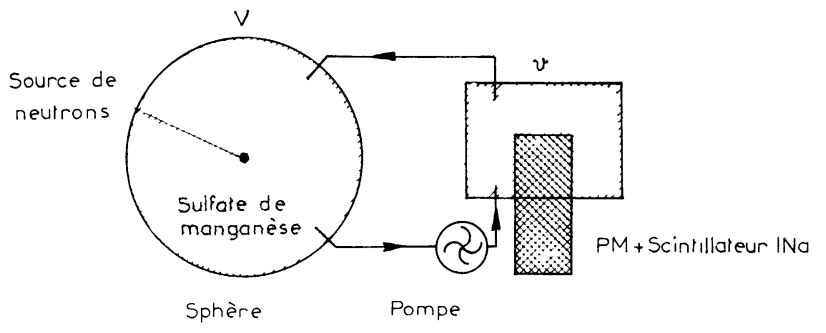

FIG. 3.

mesurer est placée dans un récipient de grandes dimensions rempli d'une solution aqueuse de sulfate de manganèse. Dans le cas d'une solution saturée, $86 \%$ des neutrons sont capturés par le manganèse et le reste par l'eau.

A l'équilibre, il y a 86 gammas pour 100 neutrons émis. Il suffit de mesurer l'activité $\gamma$ d'une fraction $\frac{v}{V}$ $\mathrm{du}$ volume total de la solution. Nous avons ainsi mesuré des sources d'activité inférieure à $10^{3} \mathrm{n} / \mathrm{s}$. Par ailleurs, les dimensions du récipient n'étant pas infinies, nous avons tenu compte de la proportion de neutrons de fuite, en mesurant avec un compteur Mac Kibben l'émission de la source à l'intérieur et à l'extérieur du compteur à activation. Le rapport des taux de comptage donne le pourcentage de neutrons de fuite avec une précision relative de $10 \%$, suffisante à l'évaluation d'un terme de correction lui-même de $10 \%$ environ.

Nous avons obtenu les activités suivantes au moment des expériences :

$$
\begin{gathered}
\text { Source PoF }=1,49 \times 10^{4} \mathrm{n} / \mathrm{s} \\
\text { Source } \operatorname{SbBe}=5,29 \times 10^{6} \mathrm{n} / \mathrm{s} \\
\text { Source PoBe no } 1=8,78 \times 10^{4} \mathrm{n} / \mathrm{s} \\
\text { Source PoBe } \mathrm{n}^{0} 2=2,97 \times 10^{4} \mathrm{n} / \mathrm{s} .
\end{gathered}
$$

C. Étalonnage par intégration graphique du flux (fig. 1). - Pour disposer d'un contrôle supplémentaire, nous avons exploité les résultats de l'expérience en géométrie infinie pour en déduire, par deux méthodes différentes, l'activité de la source PoBe no 1 et comparer la valeur obtenue à celle donnée par la méthode d'activation au manganèse $S=8,78 \times 10^{4} \mathrm{n} / \mathrm{s}$.

Une première méthode, exposée dans un autre travail [10], utilisant une expression du flux pour une source d'activité $1 \mathrm{n} / \mathrm{s}$, donne une même valeur de l'activité de la source grâce au facteur de normalisation $S: \varphi_{\exp }=S . \varphi_{\text {theor }}$
La deuxième méthode est purement expérimentale et donne la valeur de $S$ par intégration graphique de la courbe $4 \pi r^{2} n$ de la figure 1. En effet, la densité de ralentissement $q(r)$ au niveau thermique représente le nombre de neutrons qui franchissent le niveau thermique au point $(r)$. Comme le nombre de neutrons capturés en cours de ralentissement est négligeable, on peut écrire que le nombre total de neutrons qui franchissent le niveau thermique, dans tout le volume d'eau entourant la source, est égal à son activité, soit :

$$
\int_{0}^{\infty} 4 \pi r^{2} q(r) \mathrm{d} r=S
$$

D'autre part, en appelant $N(r)$ la densité des neutrons thermiques, $\varphi(r)$ leur flux, $\tau$ leur temps de vie $\left(\tau=2,10 \times 10^{-4} \mathrm{~s}\right)$, $v$ leur vitesse $(v=2,20 \times$ $\left.10^{5} \mathrm{~cm} / \mathrm{s}\right)$, en notant $n(r)$ le taux de comptage du détecteur LGT $01 \mathrm{NE} \mathrm{3/1} \mathrm{dont} \mathrm{nous} \mathrm{avons} \mathrm{calculé}$ l'efficacité exacte par rapport au flux non perturbé, soit : $\varepsilon=0,089\left(\mathrm{c} / \mathrm{s}\right.$ par $\left.\mathrm{n} / \mathrm{cm}^{2} \mathrm{~s}\right)$, on peut alors écrire successivement :

$$
\begin{gathered}
\int_{0}^{\infty} 4 \pi r^{2} \mathcal{N}(r)=\tau \int_{0}^{\infty} 4 \pi r^{2} q(r) \mathrm{d} r=\tau . S \\
\varphi(r)=v \cdot N(r) \\
n(r)=\varepsilon \varphi(r) \\
\int_{0}^{\infty} 4 \pi r^{2} n(r) \cdot \mathrm{d} r=\varepsilon \cdot v \cdot \tau \cdot S
\end{gathered}
$$

soit :

$\int_{0}^{\infty} 4 r^{2} n(r) . \mathrm{d} r=4,11 \times S . \mathrm{cps} / \mathrm{s}$ dans tout le volume.

On trouve ainsi $S=8,60 \times 10^{4} \mathrm{n} / \mathrm{s}$ en bon accord avec les résultats précédents.

La valeur de l'activité de la source ainsi trouvée ne dépend pas de la température du milieu ralentisseur. En effet, quand la température varie, le flux varie comme $v$ et la section efficace du bore comme $1 / v$ (cette remarque est aussi valable pour notre compteur).

2. ÉTUdE DU GOMPTEUR AVEG LES DIFFÉRENTES SOURGES. - A. Source de neutrons de $14 \mathrm{MeV}$. - Nous utilisons un accélérateur électrostatique de $150 \mathrm{keV}$ pour produire la réaction ${ }_{1}^{3} \mathrm{H}(\mathrm{d}, \mathrm{n}){ }_{2}^{4} \mathrm{He}$. On peut dénombrer les neutrons formés par comptage de la particule associée au moyen d'un détecteur à jonction.

Le dispositif expérimental est indiqué sur la figure 4. Le tube de sortie de l'accélérateur doit contenir le détecteur à jonction et être introduit dans le canal du compteur à neutrons qui a un diamètre de $25 \mathrm{~mm}$. Ceci nous oblige à adopter la géométrie représentée figure 4 . On remarque, en particulier, que le tube porte-cible est excentré par rapport au faisceau, pour y placer la diode détectrice. Le faisceau est délimité par deux diaphragmes minces en tantale; 


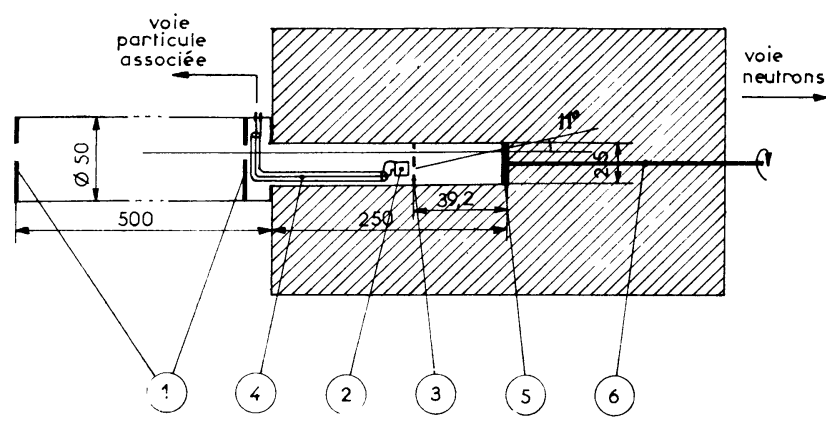

Fig. 4. - 1. Diaphragmes en tantale. -2 . Diode détectrice. - 3. Diaphragme percé de deux trous : l'un pour le passage du faisceau : $\varnothing 3 \mathrm{~mm}$, l'autre devant la diode : $\varnothing 2 \mathrm{~mm}$. - 4. Câble coaxial $50 \Omega$. -5 . Cible. - 6. Tige de commande de rotation de la cible.

on tient compte du fond de neutrons (d, d) dû aux diaphragmes, il est inférieur à $1 \%$. Nous avons obtenu les résultats suivants : efficacité totale 10,4\%, rapport des taux de comptage de la couronne extérieure et de la couronne intérieure égal à 1,19 pour des neutrons de $14 \mathrm{MeV}$.

B. Source de neutrons de 2,4 MeV. - Nous utilisons le même dispositif expérimental pour produire des neutrons par réaction $(\mathrm{d}, \mathrm{d})$. Pour évaluer le fond parasite, la cible est constituée par un disque de cuivre de $2 \mathrm{~cm}$ de diamètre recouvert de deutérium sur une moitié de sa surface seulement. On peut présenter au faisceau soit la partie deutérée, soit la surface de cuivre, au moyen d'une tige de commande traversant le compteur (fig. 4). Les résultats des mesures sont représentés sur la figure 5 : les neutrons parasites produits par réaction $(\mathrm{d}, \mathrm{d})$ sur les diaphragmes constituent un fond constant de valeur $b$, si l'intensité du faisceau reste constante $(100 \mu \mathrm{A})$, auquel ne correspond pas de particule associée. On a le nombre de neutrons $N$ détectés par le compteur qui est tel que :

$$
N=a n+b
$$

avec $n$ correspondant à la particule associée, $a$ étant un coefficient constant.

On fait varier $n$ en présentant au faisceau soit la partie active, soit la partie inactive de la cible ou en modifiant le courant reçu sur cible $(\sim 1 \mu \mathrm{A})$ par décentrage du faisceau sans modifier son intensité de $100 \mu \mathrm{A}$ pour assurer la constance de $b$.

On vérifie, sur la figure 5 , le bon accord de trois expériences successives : les points sont parfaitement alignés et le comptage des deux couronnes apparaît comme la somme des comptages des deux couronnes mesurés isolément. Le fond dû aux neutrons parasites est de l'ordre de $20000 / \mathrm{s}$.

En fait, nous avons dénombré, sur la voie de la particule associée, le nombre de protons émis par la réaction $\mathrm{d}(\mathrm{d}, \mathrm{p}) \mathrm{H}_{1}^{3}$, nous en avons déduit le nombre de neutrons produits en utilisant les résultats de la bibliographie qui relient les nombres de protons et de neutrons [9] pour une énergie de $150 \mathrm{keV}$, et un angle de $169^{\circ}$ dans le laboratoire. Dans ces conditions, il y a 6220 neutrons produits dans les $4 \pi$ pour un proton détecté sur la jonction. Les résultats de ce

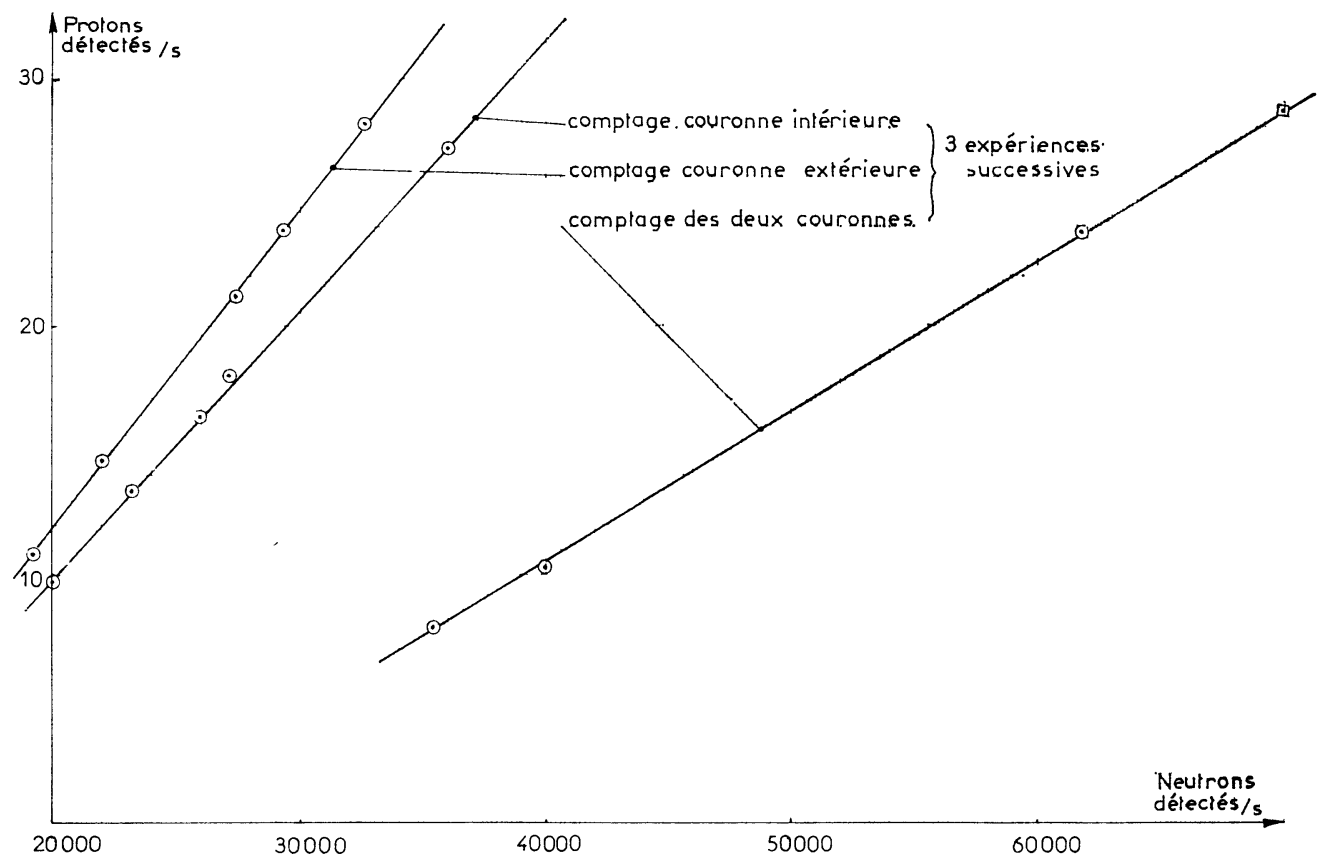

FIG. 5. - Variation du nombre de neutrons détectés par le compteur en fonction du nombre de protons détectés par la diode. 


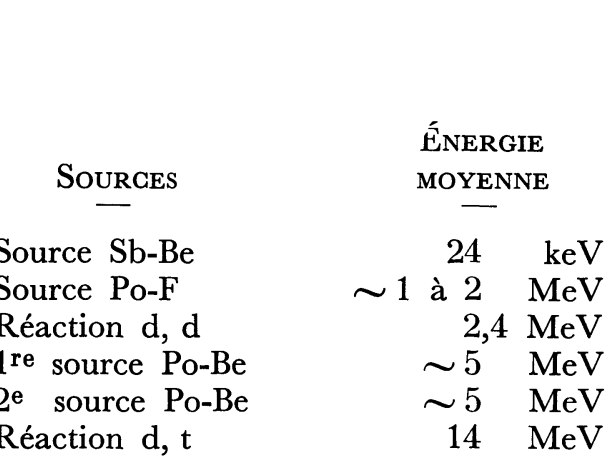

\section{EFficaGité totale}

EXPÉRIMENTALE THÉORIQUE

$30 \%$
$29,4 \%$
$27,6 \%$
$23,0 \%$
$22,2 \%$
$10,4 \%$

RAPPORT DES GOMPTAGES : couronne extérieure couronne intérieure EXPÉRIMENTAL THÉORIQUE

$\begin{array}{cl}0,43 & 0,47 \\ 0,780 & 0,78 \\ 0,89 \pm 0,02 & 0,87 \\ 0,947 & 0,96 \\ 0,962 & 0,975 \\ 1,19 \pm 0,02 & 1,17\end{array}$

différentes mesures nous donnent une efficacité totale égale à $27,6 \%$ et un rapport de comptage $\frac{n_{\mathrm{e}}}{n_{\mathrm{i}}}=0,89$
pour des neutrons de $2,4 \mathrm{MeV}$.

C. Autres sources. - Les sources polonium-béryllium et polonium-fluor déjà étalonnées sont placées successivement dans le compteur : les résultats sont consignés dans le tableau ci-dessus. En ce qui concerne la source antimoine-béryllium, l'évaluation de l'efficacité présente des difficultés en raison de sa haute activité neutronique et surtout gamma.

Ces résultats sont portés sur la figure 6 où sont représentées également les courbes théoriques obtenues dans un autre travail [10]. Nous avons trouvé des rapports et des efficacités cohérents pour deux sources Po-Be d'activités différentes.

Le compteur n'a pas une réponse constante dans la bande d'énergie entre 0 et $14 \mathrm{MeV}$, qui contient la plupart des spectres énergétiques des sources usuelles, puisque son efficacité varie entre 30 et $10 \%$. Un comptage donne donc peu de précision sur l'activité réelle de la source. Il est cependant possible d'avoir une valeur approximative de l'énergie du rayonnement, pourvu que le spectre ne soit pas trop complexe, en mesurant le rapport des taux de comptage entre les deux couronnes. Connaissant cette énergie, on en déduit l'efficacité réelle du compteur pour la source utilisée.

Conclusion. - L'étude du compteur de neutrons à efficacité définie, qui vient d'être envisagée, est basée sur le fait que l'on peut caractériser une source monoénergétique par le rapport des taux de comptage de la couronne extérieure et de la couronne intérieure qui détermine son énergie, donc l'efficacité du compteur. Cette opération est possible parce que, comme l'indique la courbe 2 de la figure 6, ce rapport

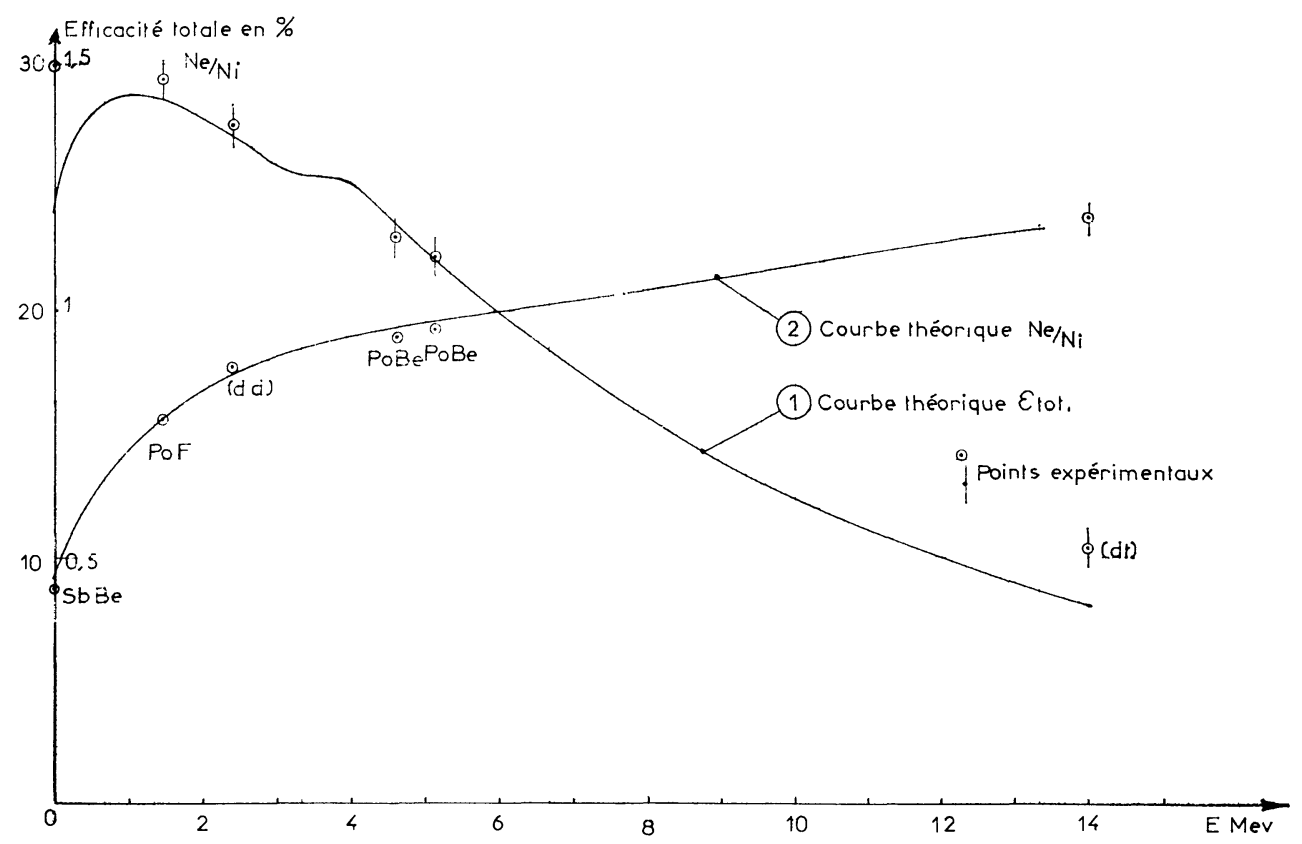

FIG. 6. - Variation de l'efficacité totale du compteur $\varepsilon_{\text {totale }}$ et de $\frac{N_{\mathrm{\theta}}}{N_{\mathrm{i}}} \frac{\text { comptage sur la couronne extérieure }}{\text { comptage sur la couronne intérieure }}$ en fonction de l'énergie. 
est une fonction croissante de l'énergie, ce qui fait qu'il ne peut pas prendre la même valeur pour des sources monoénergétiques d'énergie différente.

Dans le cas d'une source de spectre d'énergie quelconque, on peut encore définir le rapport des taux de comptage des deux couronnes, ce rapport déterminant, comme précédemment, une certaine énergie. Nous allons examiner si l'énergie ainsi obtenue correspond à l'efficacité réelle du compteur. Il est plus simple et équivalent, pour mener l'étude, d'utiliser les courbes de variation $\varepsilon_{\theta}$ et $\varepsilon_{\mathrm{i}}$ des couronnes extérieure et intérieure en fonction de l'énergie. On les déduit immédiatement de $\varepsilon_{\text {totale }}(E)=\varepsilon_{\theta}(E)+\varepsilon_{\mathrm{i}}(E)$ et de $\rho(E)=\frac{\varepsilon_{\theta}(E)}{\varepsilon_{\mathrm{i}}(E)}$.

Considérons deux sources, notées 1 et 2 , dont la somme des activités $\alpha_{1}$ et $\alpha_{2}=1-\alpha_{1}$ est de $1 \mathrm{n} / \mathrm{s}$; leurs énergies sont $E_{1}$ et $E_{2}$. Le taux de comptage $n_{\mathrm{e}_{1}^{\prime}}$ de la couronne extérieure pour la source 1 s'écrit :

$$
n_{\mathrm{e}_{1}}=\varepsilon_{\mathrm{e}}\left(E_{1}\right) \cdot \alpha_{1}
$$

on a de même pour les taux de comptage de chaque couronne et pour chaque source :

$$
\begin{gathered}
n_{\mathrm{e}_{2}}=\varepsilon_{\theta}\left(E_{2}\right) \cdot \alpha_{2} \quad \text { et } \quad n_{\mathrm{i}_{1}}=\varepsilon_{\mathrm{i}}\left(E_{1}\right) \cdot \alpha_{1} \\
n_{\mathrm{i}_{2}}=\varepsilon_{\mathrm{i}}\left(E_{2}\right) \cdot \alpha_{2}
\end{gathered}
$$

l'indice i désignant la couronne intérieure. Si on place les deux sources ensemble dans le compteur, l'efficacité de la couronne extérieure s'écrit :

$\varepsilon_{\mathrm{\theta}}\left(E_{\mathrm{e}}\right)=n_{\mathrm{e}}=n_{\mathrm{e}_{1}}+n_{\mathrm{e}_{\mathrm{a}}}=\alpha_{1} \varepsilon_{\mathrm{e}}\left(E_{1}\right)+\left(1-\alpha_{1}\right) \varepsilon_{\mathrm{e}}\left(E_{2}\right)$.

Cette relation définit une énergie $E_{\theta}$ par la courbe donnant la variation d'efficacité de la couronne extérieure en fonction de l'énergie. De même, on peut écrire, pour l'efficacité de la couronne intérieure :

$$
\varepsilon_{\mathrm{i}}\left(E_{\mathrm{i}}\right)=\alpha_{1} \varepsilon_{\mathrm{i}}\left(E_{1}\right)+\left(1-\alpha_{1}\right) \varepsilon_{\mathrm{i}}\left(E_{2}\right) .
$$

Cette relation définit encore une énergie $E_{\mathrm{i}}$. En général, $E_{\mathrm{i}}$ est différente de $E_{\mathrm{e}}$. Cherchons la condition pour que $E_{\mathrm{i}}=E_{\mathrm{e}}=E$ qui, seule, pourra avoir une interprétation physique. On doit avoir :

$$
\begin{aligned}
& \varepsilon_{\mathrm{e}}(E)=\alpha_{1} \varepsilon_{\mathrm{e}}\left(E_{1}\right)+\left(1-\alpha_{1}\right) \varepsilon_{\mathrm{e}}\left(E_{2}\right) \\
& \varepsilon_{\mathrm{i}}(E)=\alpha_{1} \varepsilon_{\mathrm{i}}\left(E_{1}\right)+\left(1-\alpha_{1}\right) \varepsilon_{\mathrm{i}}\left(E_{2}\right) .
\end{aligned}
$$

Ceci nous définit une relation fonctionnelle entre $\varepsilon_{\mathrm{e}}$ et $\varepsilon_{\mathrm{i}}$; si on l'écrit sous forme d'un polynôme, on démontre facilement que l'on a la condition :

$\varepsilon_{\mathrm{e}}(E)=A+B \varepsilon_{\mathrm{i}}(E) ; \quad A$ et $B$ étant des constantes.

Dans le cas particulier où les efficacités de chaque couronne varient linéairement en fonction de l'énergie, l'énergie $E$ définie précédemment est égale à l'énergie moyenne de la source. Notre compteur est assez éloigné de ces conditions idéales, en particulier, l'efficacité extérieure passe par un maximum. Néanmoins, il permet de mesurer l'activité de la plupart des sources neutroniques usuelles avec une précision satisfaisante. On pourrait prévoir, par un calcul théorique, un arrangement géométrique des détecteurs qui remplirait les conditions définies par la relation précédente.

Remerciements. - Nous sommes heureux de remercier MM. Ambrosino, directeur du Laboratoire de Physique des rayons X, et Felden, directeur du Laboratoire de Physique Théorique et Nucléaire de la Faculté des Sciences de Nancy, pour l'attention qu'ils ont portée à ce travail et les conseils qu'ils nous ont donnés au cours de sa réalisation.

Nous témoignons également notre gratitude au G.E.A. pour l'aide matérielle qu'il a bien voulu accorder à la poursuite de nos expériences.

$$
\text { Manuscrit reçu le } 1^{\text {er }} \text { août } 1966 .
$$

\section{BIBLIOGRAPHIE}

[1] McKibben (J. L.) et Hansen (A. O.), Phys. Rev., 1947, 72, 673 .

Ladu, Pelliccioni et Rotondi (E.), Nucl. Inst. Meth., 1965, 173-174.

Marion (J. B.), Levesque (R. J. A.), LUdeMANN (C. A.) et DETENBECK (R. W.), Nucl. Inst. Meth., 1966, 297-304.

[2] Paissner (H.) et al., Nucl. Inst. Meth., 1963, 289. GrbBons et al., Phys. Rev., 1961, 122-1, 182-210. BolLINGER et Thomas, Rev. Sci. Inst., 1957, 489, et 1962, 137.

[3] Colvin (D. W.) et SoverLy (M. G.), The accurate measurements of $\nu$ by the Boron-pile, Not for publication A.E.R.E., 1963.

[4] ALLEN (W. D.), Neutron Detection, 213.
[5] BeckurTz et WirTz, Neutron Physics, 105.

[6] Mina (H.) et Tobayama (T.), Nucl. Electronic, tome 2, Conference Proceeding Belgrade, 241.

[7] Gandy (A.), Préparation et étalonnage des sources radioactives de référence I.A.E.A., Monographie 14, 85 .

[8] HARDEN (T. B. D.), Journal of nuclear energy, 1965, 19, 607.

[9] Theuss (R. B.), McGarry (W. I.) et Beach (L. A.), Nuclear Physics, 1966, 273-289.

[10] LE BARS, PICOT, Étude théorique de l'atténuation en fonction de la distance, du flux thermique produit par une source ponctuelle monoénergétique de neutrons rapides, en milieu hydrogéné (en cours de publication).

Le Directeur de la Publication : Jules-Jean Chovis. - Imprimerie des Presses Universitaires de France, Vendôme (France) Dépôt légal : 3-1967 